\title{
Assessment of Health Management Information System (HMIS) implementation in South West Shewa Zone, Oromia, Central Ethiopia.
}

Addisuu Bogale ( $\sim$ addisuub@gmail.com )

Pathfinder International Ethiopia https://orcid.org/0000-0002-1084-1300

\section{Dufera Rikitu}

Wollega University

\section{Research Article}

Keywords: Assessment, HMIS, Implementation, Ethiopia

Posted Date: October 6th, 2020

DOI: https://doi.org/10.21203/rs.3.rs-86339/v1

License: (c) (1) This work is licensed under a Creative Commons Attribution 4.0 International License.

Read Full License 


\section{Title:}

Assessment of Health Management Information System (HMIS) implementation in South West Shewa Zone, Oromia, Central Ethiopia.

\section{Author:}

Addisu Bogale

Pathfinder International Ethiopia, Addis Ababa, Ethiopia.

Corresponding author

Full name: Addisu Bogale

Tel. (+251) 910107254

E-mail: addisuub@gmail.com 


\begin{abstract}
Background: Health management information system (HMIS) is a system that allows for the collection, storage, compilation, transmission, analysis and usage of health data that assist decision makers and stakeholders manage and plan resources at every level of health service. The objective was to assess Health Management Information System (HMIS) implementation in south west shewa, oromia, central Ethiopia 2019.

Methods and Materials: The assessment was conducted in south west Shewa from August 17 to 25, 2019. Facility based cross sectional study design was employed and 22 health centers were included in the study. Collected data was entered in Epi-data and analyzed using SPSS 23 and Microsoft excel 2010.

Results: Among health centers assessed 58\% and 92\% had HMIS unit and electric power respectively. In all woredas HMIS focal person was assigned and trained on information use. At health center level HMIS recording and reporting, indicator reference, NCoD and information use manuals availability were $25 \%$, $33 \%, 17 \%$ and 58\% respectively. The study shows that implementation of information display, and functionality of PMT were $74 \%$ and 58\% respectively. During the assessment interviewed midwives on the service delivery unit only $75 \%$ of health center define and record new and repeat acceptor of family planning according to the national revised HMIS indicators. Also 67\% of health officer assigned to outpatient department define new and repeat and filled in the register properly and some of them didn't use NCoD to classify HMIS disease classification.

Conclusions: Health management information System (HMIS) in south west shewa zone was not fully implemented and there is a challenge related to improving data accuracy, access to computerized HMIS data and competencies to analyze, interpret and use of HMIS data at health center levels. Also, presence of reporting mechanism, displaying information and feedback mechanism were poor at health center level. Therefore, it is important to function performance monitoring team meeting and supportive supervision regularly at all levels.
\end{abstract}

Keywords: Assessment, HMIS, Implementation, Ethiopia 


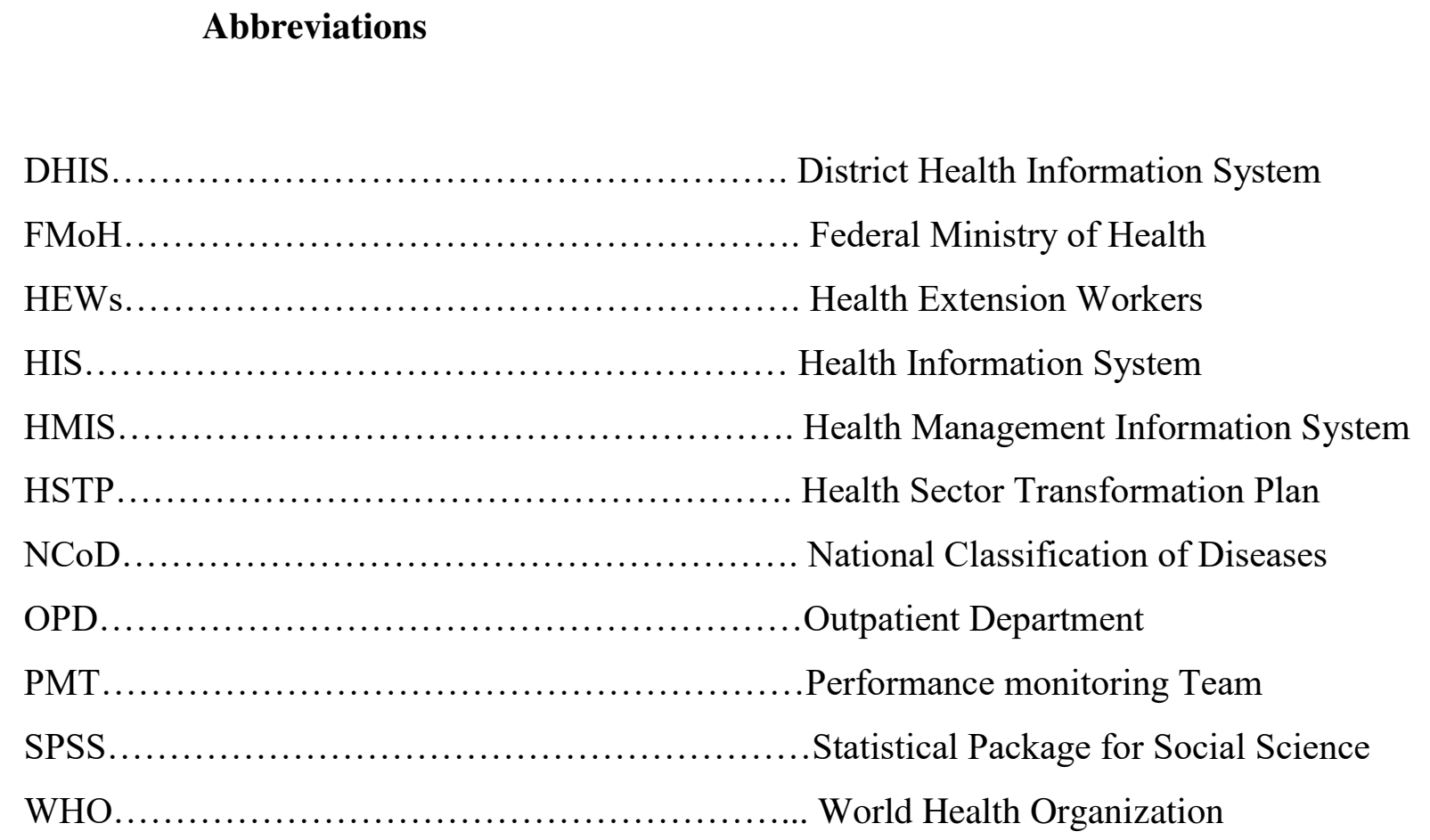




\section{INTRODUCTION}

Health Management Information System (HMIS) is a system that allows for the collection, storage, compilation, transmission, analysis and usage of health data that assist decision makers and stakeholders manage and plan resources at every level of health service (1). Health information is the processed data and knowledge that an individual or group use to support their decisions in the health sector $(1,2)$. It is fundamental for the overall health system which informs decision-making in each of the other five blocks of the system and improving managerial decisions by providing quality information for evidence-based health practices. The World Health Organization (WHO)'s framework for health systems strengthening identifies six attributes of a health system (2). The attributes, or building blocks, include a health workforce; health services; health financing; governance and leadership; medical products, vaccines, and technologies; and health information. While each building block of the WHO framework is important to improving health systems and ultimately health outcomes, quality and timely data from health information systems (HIS) are the foundation of the overall system and inform decision making in each of the other five building blocks in the health system (3).

Routine health information is vital for operational, tactical, and strategic decision-making. Major problems in relation to health information which was identified by the World Health Organization (WHO) are inadequate use of existing information and evidences due to fragmentation and duplication of health information(4). In line with this the participants of the Global Summit on Measurement and Accountability for Health identified a five-point call to action with a set of targets for better data use in support of healthrelated sustainable development goals (SDG). One of this five-point call to action is "by 2020, countries have health information flows that involve the use of data locally to improve services and programs" $(5,10)$.

Public health decision-making is seriously reliant on a timely availability of sound data, and globally significant human and financial resources have been invested to improve health information systems (6). It is well accepted that information generated by health care systems is used for planning, management of health commodities, detecting outbreaks, and monitoring the overall performance of the health system that further maintains the quality of care $(7,11)$.

Sub-Saharan African countries recognized and accepted Health Management Information System (HMIS) as a source of routine health information, however, health programs frequently fall short of its efficient use to inform decisions. Ethiopian implement Health Management Information System (HMIS) since 2008 to 
capture and provide 131 indicators (8) used to improve the provision of health services, ultimately to improve health status of the population.

The findings of the assessment will be expected to identify major problems observed in health institutions in implementing HMIS in south west shewa zone. It will provide relevant information for planning monitoring and evaluation and show areas that needs special attention and further follow up for program improvement. Furthermore, zonal health office, woreda health offices, and health center can use the finding of this assessment as an input for informed decision making in resource allocation and identifying areas needs special concern. 


\section{METHODS AND MATERIALS}

\section{Study area and period of assessment}

The study was conducted in health facility of south west shewa zone, Oromia, central Ethiopia, from August 17 to 25, 2019. Southwest Shewa zone is one of the zonal administrations located in Oromia regional state. The zonal capital, Woliso, is $114 \mathrm{~km}$ from the capital city of Ethiopia, Addis Ababa. According to a report from the zonal health office in 2018 , the total population of the zone was $1,101,129$. There are 11 woredas and one town administration, 264 rural and 22 urban kebeles in the zone. According to population projection of 2007 , there were a total of 1,101,129 populations (Male 556,194,\& Female 544,935) , and urban 149,878 and rural 951,251 residents in the zone (12). There are 4 functional government hospitals, 1 Private not for profit hospital, 54 functional health centers, and 72 different levels of private clinics and 264 health posts that serve the population living around. The study was carried out in four woredas such as Wonchi, Waliso Town, Goro and Waliso Rural. The health centers are currently implementing Health Management Information System.

\section{Study Design}

- Cross sectional study design.

\section{Source Study}

- Source of the study were all health centers found in south west shewa zone,

\section{Study units}

- The study units of the assessment were selected health centers,

\section{Inclusion criteria}

- Health centers implementing health management information system were included in the assessment. 


\section{Sample Size and sampling Procedure}

\section{Sample size determination}

According to the WHO guideline for sampling district health system for assessing its functionality we select $4(30 \%)$ of the zone woredas were taken (13). Therefore, 22 health centers were included in the assessment and all health facilities' documents from April to June 2019 were reviewed.

\section{Sampling technique}

For this assessment, at zonal level, woredas were randomly selected. After selection of woredas, health centers randomly selected using lottery method depending on the number of health centers found in each woreda. All health professionals and supporting staffs working on HMIS and those are available at the time of data collection was interviewed in the study and all selected health centers were physically observed.

\section{Data collection}

\section{Data collection Instrument}

The data was collected using HMIS structured questionnaires to answer the objective of the assessment. The questionnaire contains five components 1) Appropriateness of the card room given weight of 9 scoring points 2) Functionality of HMIS system (service delivery point, records, registers, completeness and timeliness of reports) given weight of 12 scoring points 3) Information display given weight of 4 scoring points 4) Performance Monitoring Team (PMT) given weight of 11 scoring points 5) Supportive Supervision system given weight of 8 scoring points. Finally, data quality was observed from registers, tally sheet and reports.

\section{Data Collectors}

Ten BSc health professionals who know local language and trained on HMIS were recruited for data collection. One day orientation was given for data collectors on data collection tools and procedures by the principal investigators.

\section{Data collection Field Work}

Quantitative data were collected from health professionals and supporting staffs working on HMIS and service delivery unit was interviewed using structured questionnaire. Prior to start the interview, data collectors were communicated with health centers head to obtain information about the staffs and whom to interview. Data was checked for completeness and accuracy by data collectors though out the data collection period. 


\section{Data Analysis}

The data was checked for completeness and coded by Epidata3.1 version. Microsoft Excel 2016 was used to develop the frameworks and SPSS version 23 was used for data analysis. A variety of descriptive statistics such as mean scoring, and percentage was calculated to describe the results.

\section{Data Quality management}

Questionnaire was prepared in English and translated to local language (Afan Oromo) and retranslated back into English to ensure its consistency. The questionnaires were pre-tested at Tare health center and to ensure that whether it is clear or not for other health centers and then some corrections was done accordingly. Data collectors were instructed to check the completeness of each questionnaire at the end of each interview. The completeness of the questionnaire at the end of the day was rechecked by supervisors.

\section{Ethical consideration}

Appropriate research ethical clearance was obtained from the ethical review committee of South West Shewa Zonal health office (reference number: WEFG/241/2019, July 2019) and woreda health office. This study was conducted in accordance with the Declaration of Helsinki: each study participant was well informed about the aim of the study, benefits and risks; informed written consent was secured from study participants; study participants' confidentiality was maintained; no personal identifiers were used in the data collection questionnaire and codes were used in place of them; data were kept in a protected and safe location where paper-based data were kept in a locked cabinet and computer-based data were protected using passwords; the recorded data were not accessed by a third person, except the researcher; and data sharing will be enacted based on the consent and permission of research participants and the ethical and legal rules of data sharing. 


\section{RESULTS}

\section{Overview of Woredas and Health Centers}

Total numbers of study health centers were $100 \%$ response rate. Health staffs such as HMIS focal person, and heads of health centers were interviewed during the assessment. Health centers had HMIS unit and electric power access were 58\% and 92\% respectively. All Woredas HMIS focal person was assigned and trained on DHIS2 and information use (Table1).

\section{Functionality and implementation of Card Room}

Health Information System (HMIS) implementation in the medical record room (MRU) as per standard at Dilela, Chitu, Goro and Obi health centers using maximum scoring of 9 points and MRU implementation in Dulele and Selam Gatiro health centers were poor implementation (Figure 1).

\section{Functionality of Health Management Information System}

Functionality of HMIS system was assessed and checked at service delivery point, records, registers, completeness and timeliness of reports. Functionality of Health Information System (HMIS) implementation as per guideline were almost all implemented in Waliso, Goro, Chitu and Korke health centers using maximum scoring of 12 points and Selam Gatiro health center was poor implementation (Figure 2).

\section{Performance Monitoring Team (PMT)}

From the finding, performance monitoring team (PMT) were fully functional as per guideline in Chitu and Obi health centers and total not functional in Wayu and Leman health centers. Generally, functionality of performance monitoring team (PMT) was 58\% (Figure 3). The assessment also revealed that Performance monitoring team conducting Lot quality assurance sampling (LQAS) at health facility level and Routine data quality assurance(RDQA) at woreda level were $58 \%$ and $21 \%$ respectively(Table 3 )

\section{Information Display}

Availability of tables, charts and maps on maternal health indicators, child health indicators, facility utilization, and disease surveillance indicators were assessed for understanding the level of information display in health centers. The assessment shows that health centers display information as per guideline was $41 \%$ and $2(1 \%)$ of health centers not display information (Figure 4$)$.

\section{Supportive Supervision}

Using standard checklist supportive supervision from different levels was one of the implementations of HMIS. During the assessment $9(41 \%)$ of health centers score eight and 2(1\%) of health centers not conduct any supportive supervision for the next level as per guideline (Figure 5). 


\section{Overall HMIS Implementation}

The assessment finding shows that over all HMIS implementation Information display, and Functionality of performance monitoring team (PMT) were $74 \%$ and 58\% respectively. Functionality of performance monitoring team was curial to implement HMIS as per guideline (Figure 6).

\section{Guidelines and manuals for implementation of HMIS}

At least four manuals which facilitate the implementation of HIS are in place within the Woreda Health Office and Health center level. During this assessment at woreda level all manuals are available. At health center level HMIS recording and reporting, indicator reference, $\mathrm{NCoD}$ and Data quality and information use manuals are $25 \%, 33 \%, 17 \%$ and $58 \%$ respectively (Table 2 ).

\section{Data quality and information use}

In the revised HMIS (9), definition of repeat contraceptive acceptors was modified to those clients who are ever users of any contraception and each year are coming for the first time for contraception either for resupply or restarting or starting a different method of contraception. Thus, ever-user clients who come for second and subsequent visits are not counted. Thus, there were chances that the health staff might confuse the definition of Repeat Contraceptive Acceptors. During the assessment interviewed midwives on the service delivery unit only $75 \%$ of health center define and record new and repeat acceptor according to the national standard. Also, the data elements of different registers were did not filled properly according to the national guidelines and manuals for instance Antenatal care, PNC, Delivery registration the column box of reportable data element at the end of the registration page also not filled.

On the other hand, in case of OPD attendance, the patients' data is recorded in OPD Abstract Register and in OPD Tally sheet. In the register, one row is used for one visit and the main diagnosis is recorded even if the patient comes for more than one illness. On the other hand, in the Tally sheet, every diagnosis is tallied; moreover, the tally sheet allows tallying by age and sex groups. This arrangement encourages the health staff to rely on the tally sheet for reporting and there are chances that the records in the register and tally sheet might not match. From this assessment $67 \%$ of staffs define new and repeat and filled in the register properly and some of them didn't use NCoD to classify HMIS disease classification. 


\section{DISCUSSION AND RECOMMENDATIONS}

\section{Discussions}

Among health centers assessed 58\% and 92\% had HMIS unit and electric power respectively. In all woredas HMIS focal person was assigned and trained on DHIS2 and information use. At health center level HMIS recording and reporting, indicator reference, $\mathrm{NCoD}$ and information use manuals availability were $25 \%$, $33 \%, 17 \%$ and $58 \%$ respectively. The study also reveals that implementation of information display, and functionality of PMT were 74\% and 58\% respectively. During the assessment interviewed midwives on the service delivery unit only $75 \%$ of health center define and record new and repeat acceptor of family planning according to the national revised HMIS indicators. Also $67 \%$ of health officer assigned to outpatient department define new and repeat and filled in the register properly and some of them didn't use NCoD to classify HMIS disease classification. This is almost like the study conducted in southern nation nationalities and peoples of Ethiopia (11). This is may be due to the similarity of health facility. Poor understanding of definition of indicators such as OPD visits and low capacity to calculate data were also contributing to the low level of data accuracy. Even though reports are scanned and entered into the database automatically a similarly low level of data accuracy also was observed while comparing the paper report against district health information system.

The use of information, another dimension of HMIS performance, was found limited in the assessed woreda. The revised HMIS in 2017 is geared towards supporting and strengthening local action-oriented performance monitoring (10). Health management information system using guidelines helps to identify gaps, to develop action plan and review progress continually improving service coverage over time. In the assessed health

facilities absence of such guideline may be one of the contributing factors for the observed minimum use of HMIS information in the annual plans. This finding is consistent with the limited competence in data analysis, interpretation and problem solving at the health centers. It shows data are being collected primarily for reporting and use of data for evidence-based decision making is low at peripheral level. 


\section{Recommendations}

The assessment identified strengths and weaknesses of the health management information system implementation in terms of the standards set on guidelines. Based on the findings, the following general recommendations are provided based on the findings of the assessment for further intervention for health centers, and woredas.

\section{For Health Centers}

$\checkmark$ Monitor and confirm that the health facility executes the entire health management information system procedures in respective service units to generate quality data

$\checkmark$ Compile reportable data and prepare reports timely; check report continent completeness using visual scanning; perform Lot quality assurance sampling (LQAS) with involvement of all performance monitoring team members; and then review in performance monitoring team meeting before reporting to next level

$\checkmark$ Develop performance monitoring team work plan and implement properly to monitor data quality, review performance and provide technical support to the service units or departments

$\checkmark$ Conduct regular performance monitoring tea meeting, check data quality, identify performance gaps, analyze root causes, develops action plans, implement and monitoring the progress using health management information system data

$\checkmark$ Ensure proper documentation that all-important documents such as report copies, work sheets, used registers and tally sheets are properly archived

\section{For Woredas and Zonal Health Department}

- Make sure that health workers get proper orientation and skill on health management information system through gap identification, and facilitate the provision of orientation or training

- Check report timeliness, continent \& representative completeness using report tracker and visual scanning through sharing the data to respective program and department units, and then review in performance monitoring team meeting before reporting to next level. 


\section{Acknowledgements}

I would like to express our sincerely thanks to the participants of the study and the south west shewa zonal and woreda administrative offices for their cooperation throughout the data collection process.

\section{Conflict of interest}

No author declared conflict of interest 


\section{References}

1. Wyatt JC, Sullivan F. What is health information? Bmj. 2005;331(7516):566.

2. World Health Organization (WHO). The World Health Report HEALTH SYSTEMS FINANCING. 2010

3. Winter A, Haux R, Ammenwerth E, Brigl B, Hellrung N, Jahn F. Health Information Systems. 2010;33-42.

4. FMOH. Federal Democratic Republic of Ethiopia Ministry of Health Health Sector Development Program IV October 2010 Contents. 2014;(October 2010).

5. Targets SDGH. Ensure healthy lives and promote well-being for all ages. 2016;25-35.

6. Lærum H, Karlsen TH, Faxvaag A. Use of and attitudes to a hospital information system by medical secretaries, nurses and physicians deprived of the paper-based medical record: A case report. BMC Med Inform Decis Mak. 2004;4:1-10.

7. Shaikh BT, Rabbani F. Health management information system: a tool to gauge patient satisfaction and quality of care. East Mediterr Heal J . 2005;11(1-2):192-8.

8. John Snow Inc. Health Management Information System (HMIS) - Facilitator's Guide for Training of Hospital Staff. Meas Eval. 2010;(June):1-83.

9. FMOH. Manual, HMIS Information Use and Data Quality manual. FMOH. 2014;

10. manual p. federal ministry of health policy, planning and monitoring \& evaluation directorate ( ppmed ) november 2018. 2018;(november).

11. Belay H, Azim T, Kassahun H. Assessment of Health Management Information System ( HMIS ) Performance in SNNPR, Ethiopia.

12. "The 2007 Population and Housing Census of Ethiopia: Statistical Report for Oromiya Region; "The 2007 Population and Housing Census of Ethiopia. Addis Abeba; 2007. 518 p.

13. Sambo LG, Chatora RR. Tools for Assessing the Operationality of District Health Systems. $2003 ; 10$.

14. Roadmap IR. Ethiopian Federal Ministry of Health Information Revolution Roadmap. 2016;(April).

15. Adane T. Assessment on Utilization of Health Management Information System at Public Health Centers Addis Ababa City Administrative, Ethiopia. Internet Things Cloud Comput. 2017;5(1):7. 
Table 1:Resources available for HMIS for woredas and health centers level,2019

\begin{tabular}{lcc}
\hline & Health Center & Woreda Health Offices \\
& $($ YES $)$ & $100 \%$ \\
\hline HMIS Unit & $58 \%$ & $100 \%$ \\
Have DHIS2 Computer & $75 \%$ & NA* $^{*}$ \\
Have Master Patient Index (MPI) & $41 \%$ & NA* $^{*}$ \\
Have standard Shelves & $58 \%$ & $75 \%$ \\
Functional Virtual Private Network (VPN) line & $25 \%$ & $100 \%$ \\
Local Area Network (LAN) expansion done & $42 \%$ & $100 \%$ \\
Trained Focal person on HMIS & $58 \%$ & $100 \%$ \\
Health Informatic Technician (HIT) assigned & $92 \%$ & $100 \%$ \\
Have Electric power & $92 \%$ & \\
\hline
\end{tabular}

Note: * Not Applicable

Table 2:Guidelines and Manuals for implementation of HMIS, 2019

\begin{tabular}{lcc}
\hline Manuals/Guidelines & $\begin{array}{c}\text { Woreda Health office } \\
(Y E S)\end{array}$ & $\begin{array}{c}\text { Health Center (YES) } \\
\text { HMIS Recording and reporting manual }\end{array}$ \\
HMIS Indicator reference manual & $100 \%$ & $33 \%$ \\
HMIS National Diseases Classification (NCoD) manual & $100 \%$ & $83 \%$ \\
HMIS Data Quality and Information use manual & $100 \%$ & $42 \%$ \\
CHIS User's Manual Afan Oromo Version & $100 \%$ & $21 \%$
\end{tabular}


Table 3:Establishment and Functionality of Performance Monitoring Team (PMT), 2019

Performance Monitoring Team (PMT)

Formally established PMT as national standard

PMT members participated in data quality check

PMT meeting conducted continuously

PMT meeting minutes documented

PMT minutes book clearly shows date, time, and attendees,

meeting agenda, summary of discussion $\&$ conclusions

Institution head or deputy head was chaired PMT meeting

PMT identifies performance gaps

PMT set priority to solve performance gaps

PMT identify root causes and developed action plan using problem investigation and action plan form

PMT conducted resource mapping using stakeholder analysis

PMT ensure and implement proposed interventions and started result monitoring

PMT conducting Lot Quality Assurance Sampling or Data

Quality Assurance quarterly

\section{Health Center \\ (YES)}

$75 \%$

$84 \%$

$58 \%$

$67 \%$

$75 \%$

$83 \%$

$42 \%$

$33 \%$

$33 \%$

$25 \%$

$25 \%$

$58 \%$
$25 \%$

\section{Woreda Health \\ Office (YES)}

$100 \%$

$100 \%$

$100 \%$

$100 \%$

$50 \%$

$100 \%$

$75 \%$

$50 \%$

$50 \%$

$15 \%$

$25 \%$ 


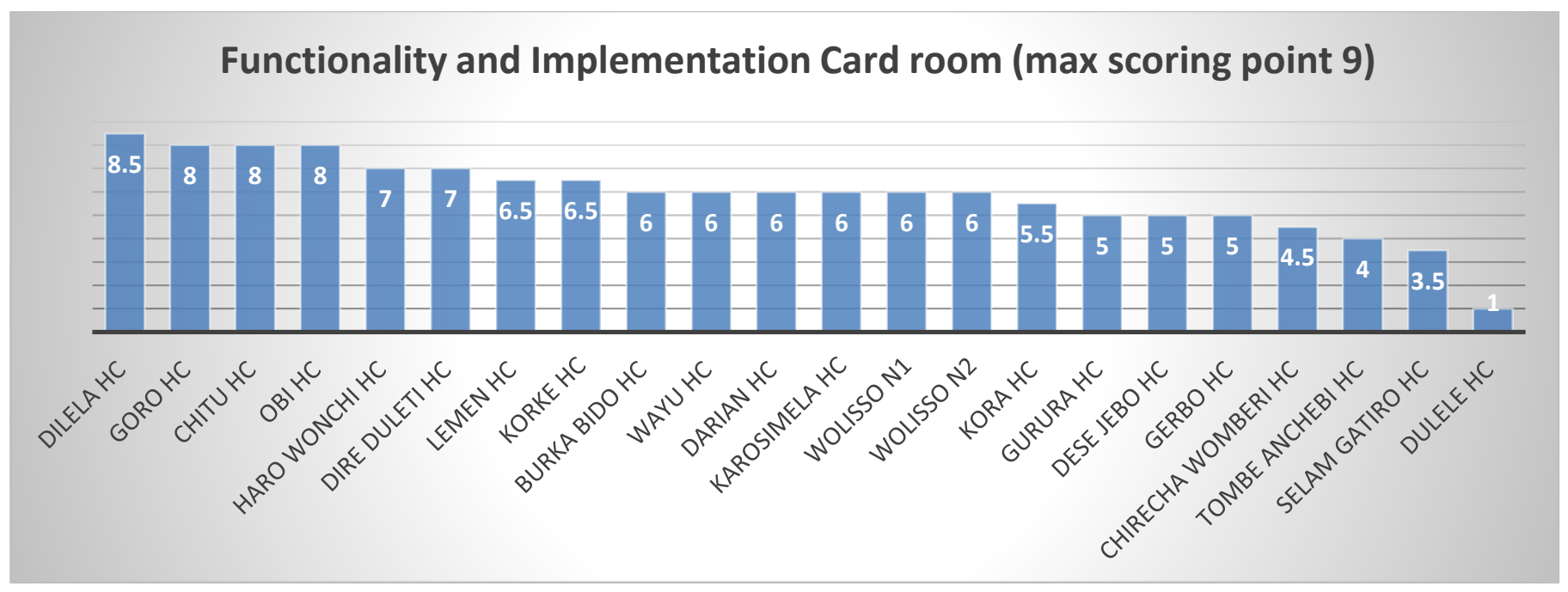

Figure 1:Functionality and Implementation of Card rooms,2019

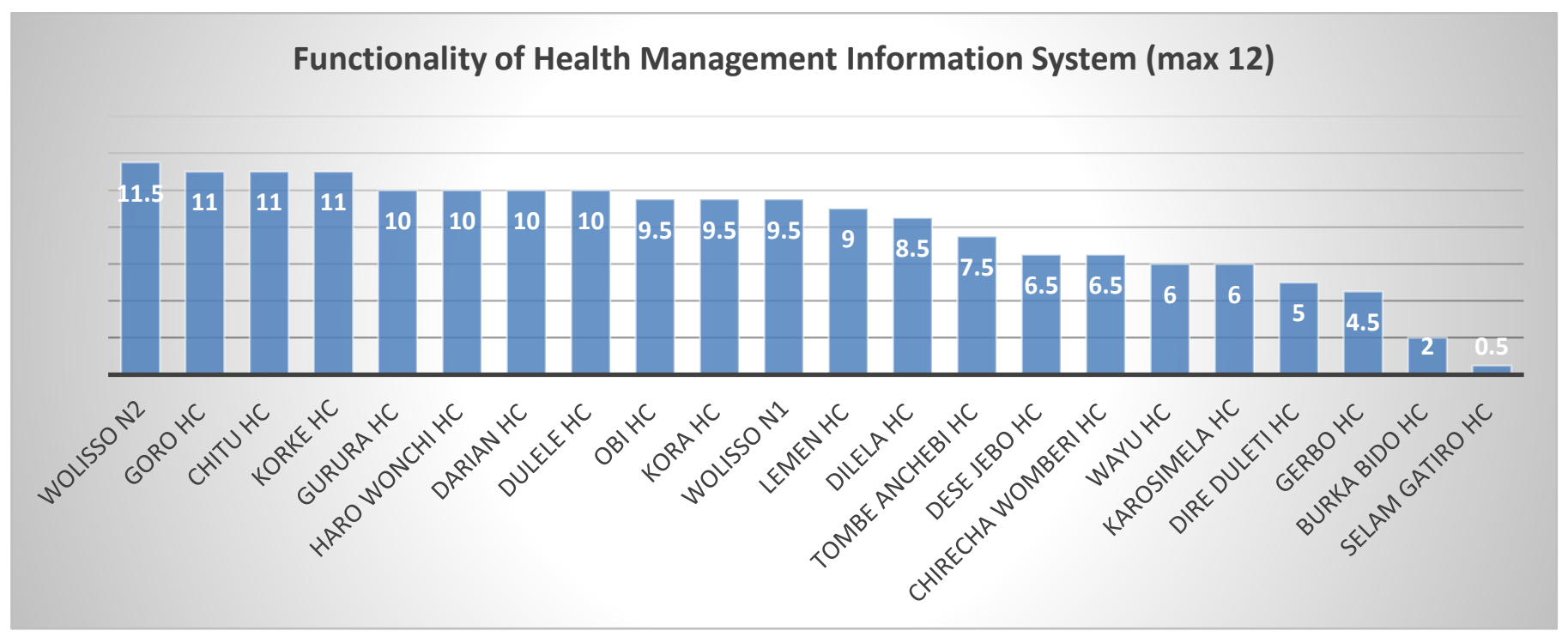

Figure 2: Functionality of Health Management Information System,2019 


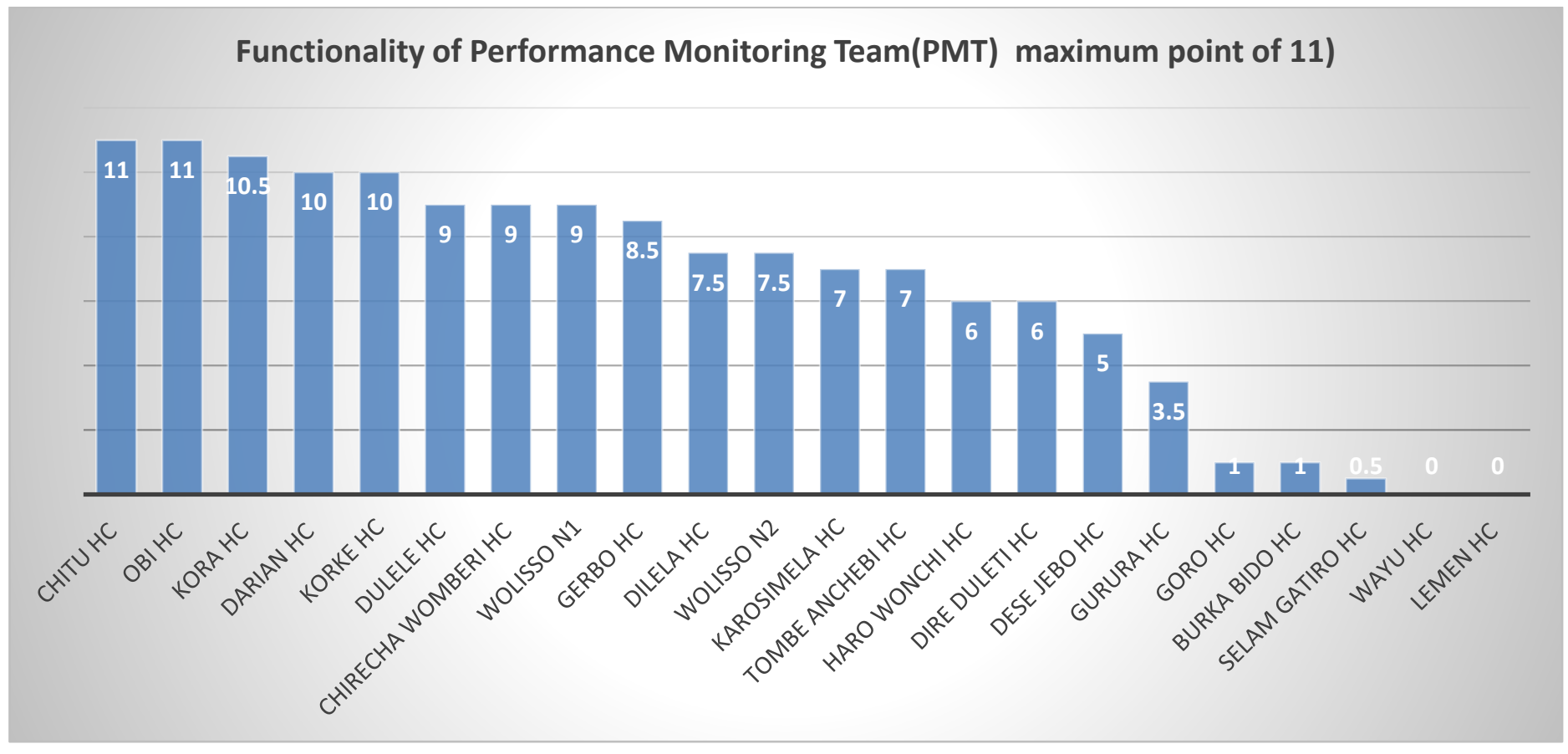

Figure 3: Establishment and Functionality of Performance Monitoring Team (PMT), 2019

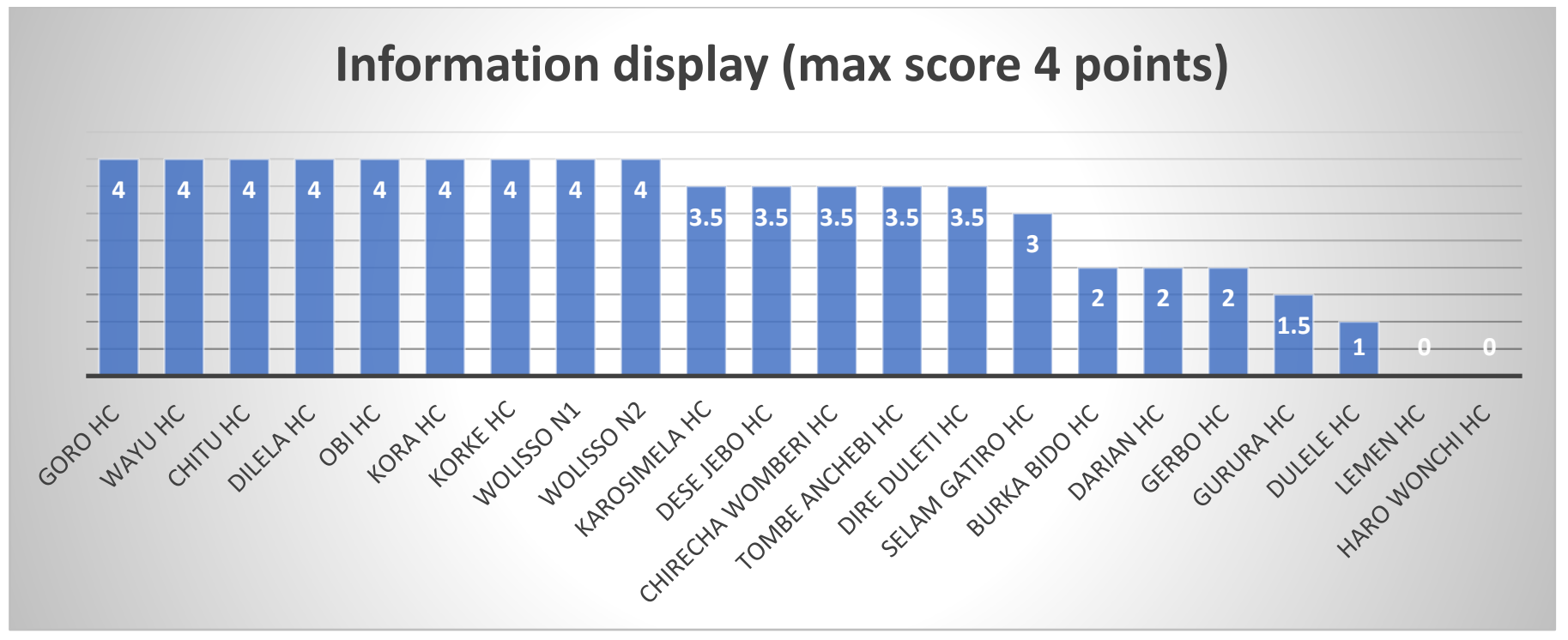

Figure 4: Displaying Information at Health Center level, 2019 


\section{Supportive supervision $(\max 8)$}

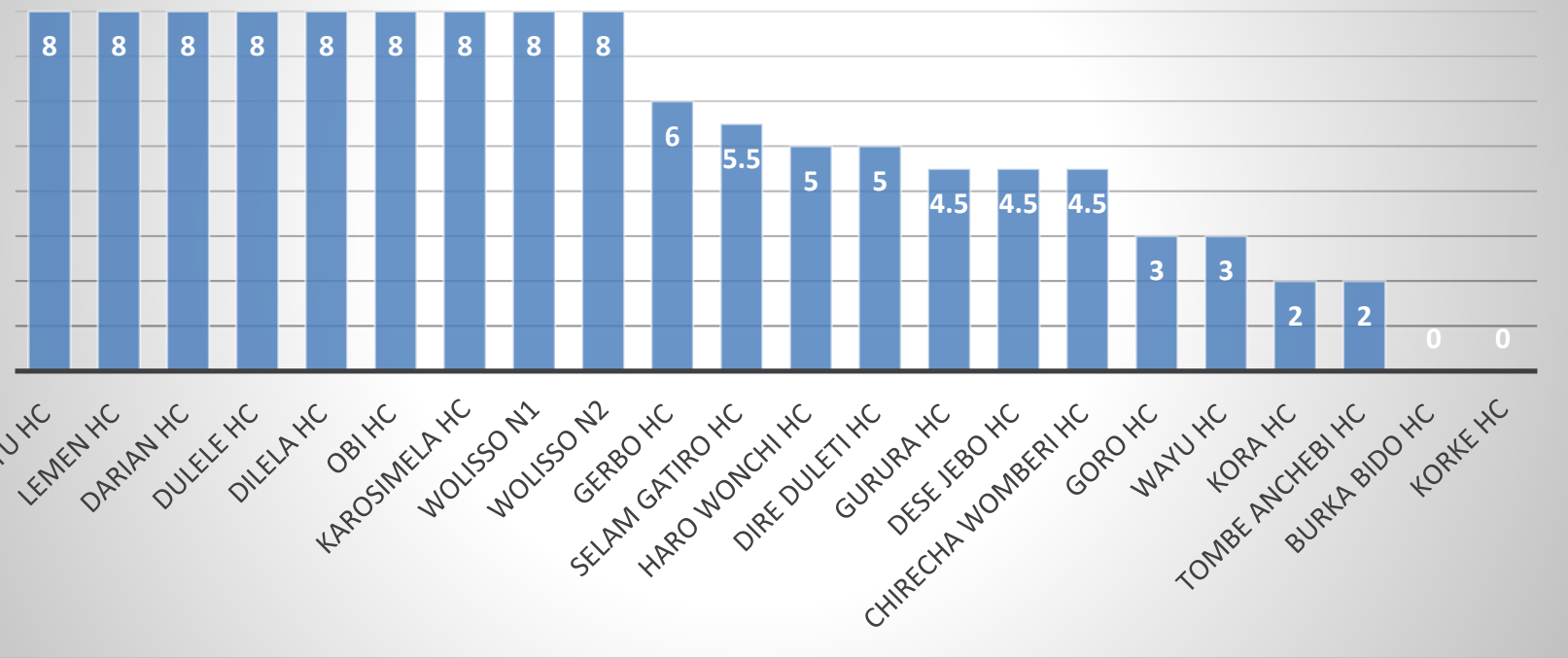

Figure 5: Supportive Supervision at Health Centers,2019

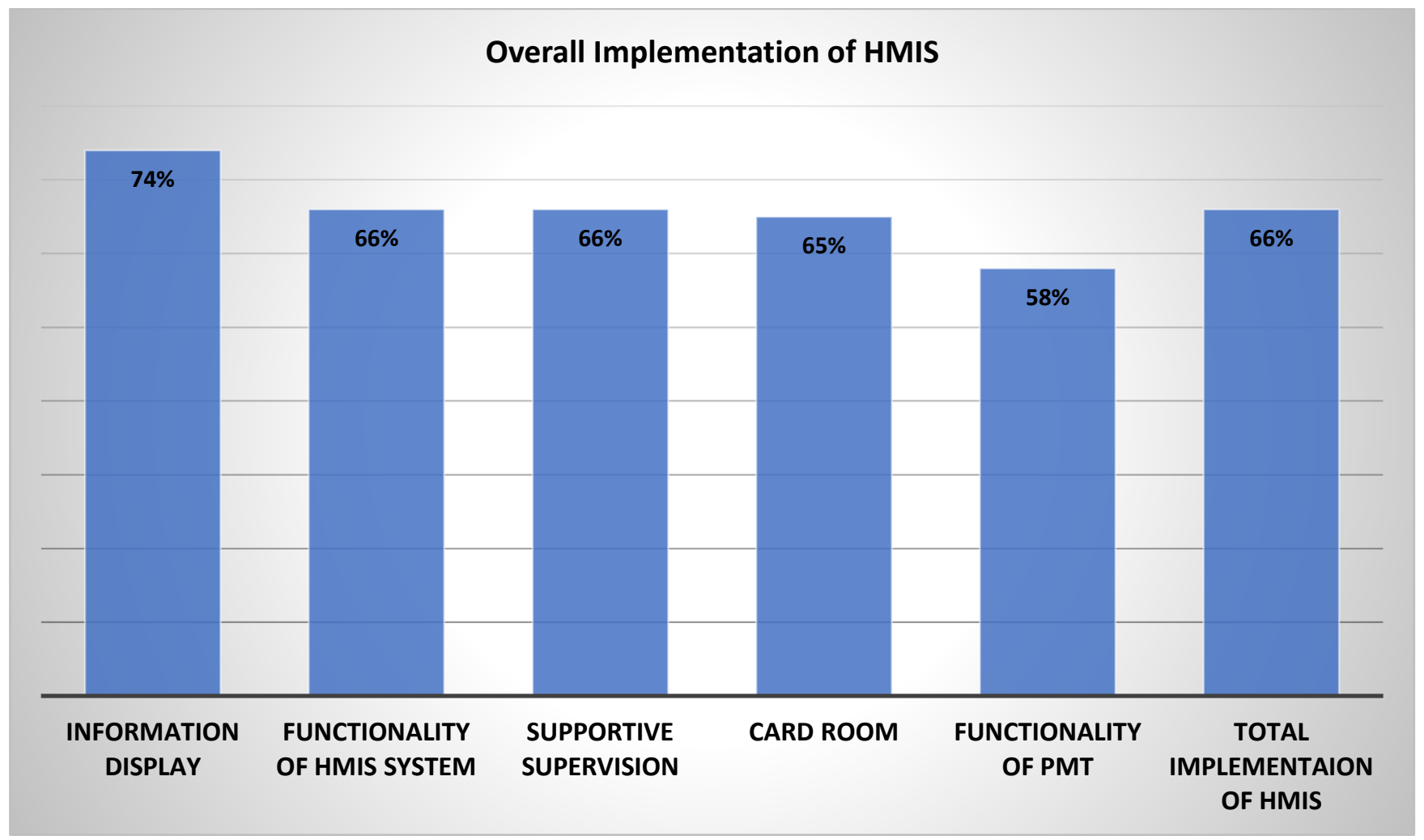

Figure 6:Overall implementation of HMIS,2019 


\section{Figures}

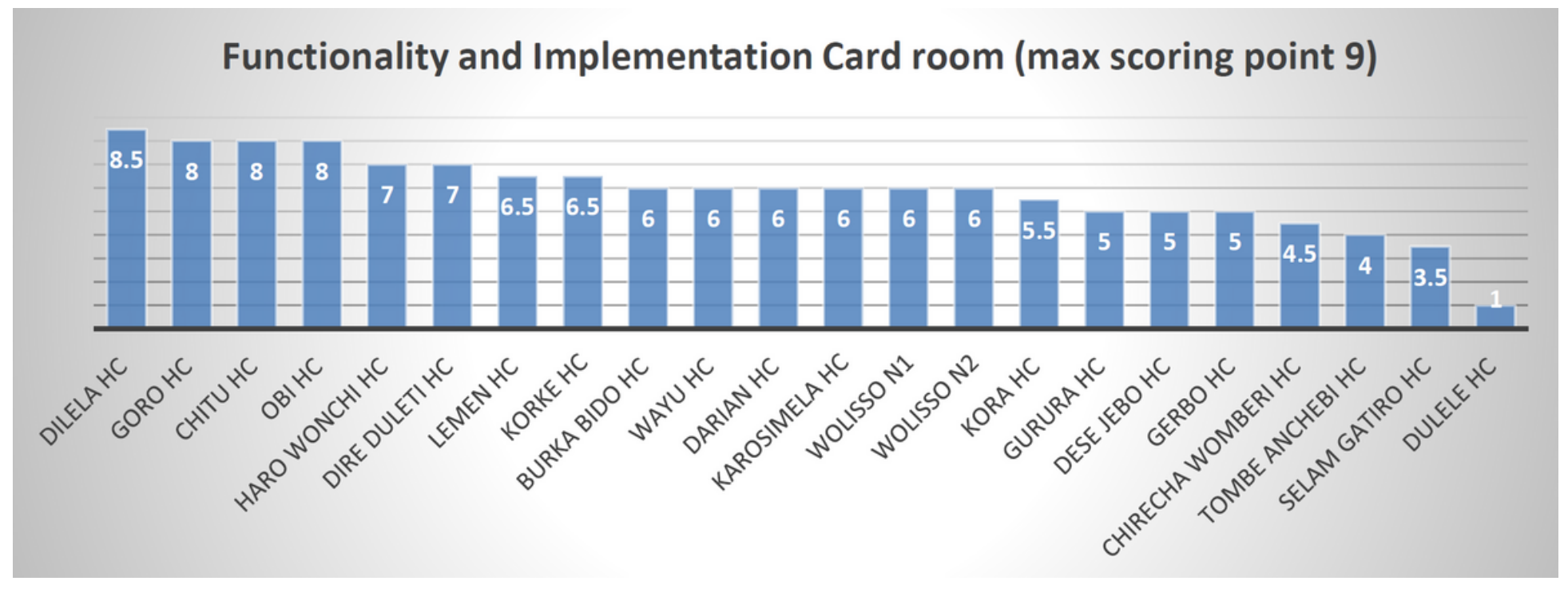

Figure 1

Functionality and Implementation of Card rooms, 2019

\section{Functionality of Health Management Information System (max 12)}
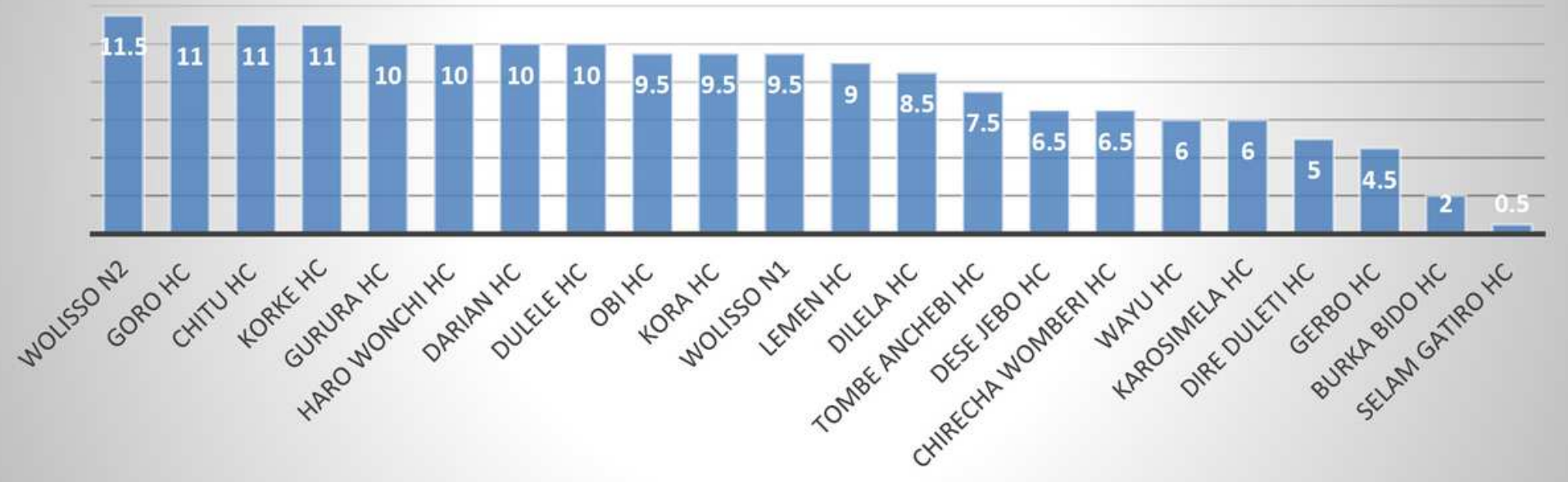

\section{Figure 2}

Functionality of Health Management Information System, 2019 


\section{Functionality of Performance Monitoring Team(PMT) maximum point of 11)}

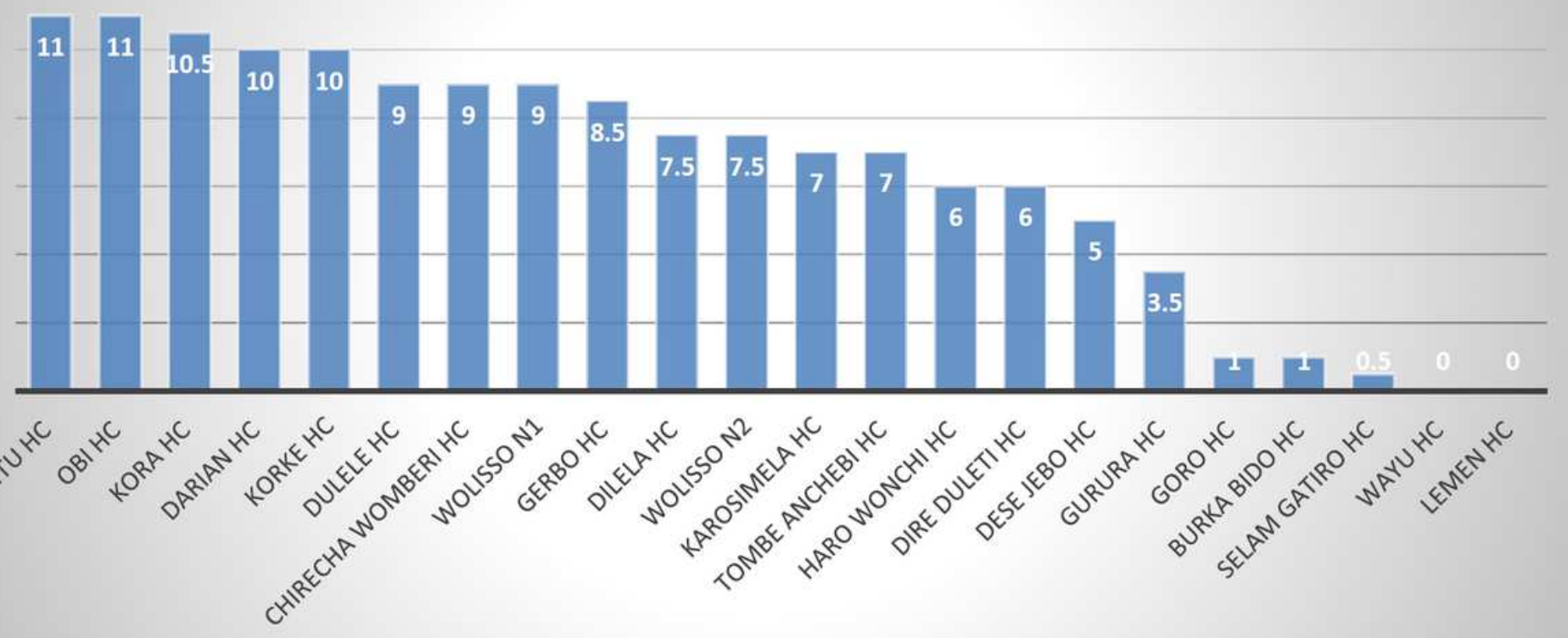

\section{Figure 3}

Establishment and Functionality of Performance Monitoring Team (PMT), 2019

\section{Information display (max score 4 points)}
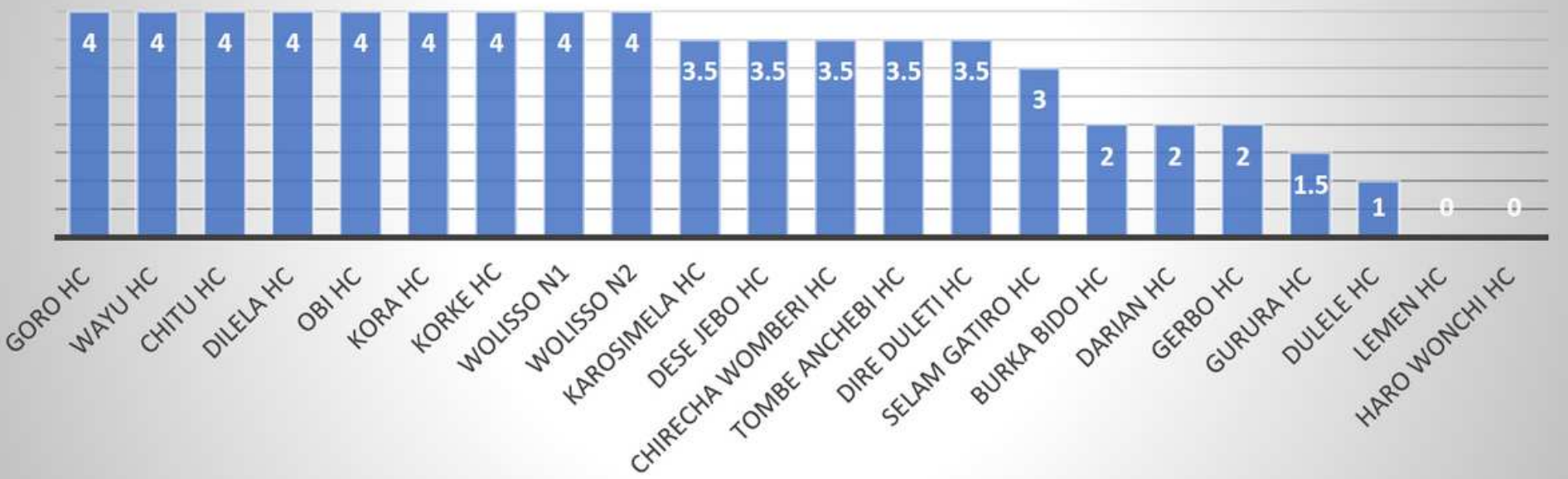

\section{Figure 4}

Displaying Information at Health Center level, 2019 


\section{Supportive supervision $(\max 8)$}

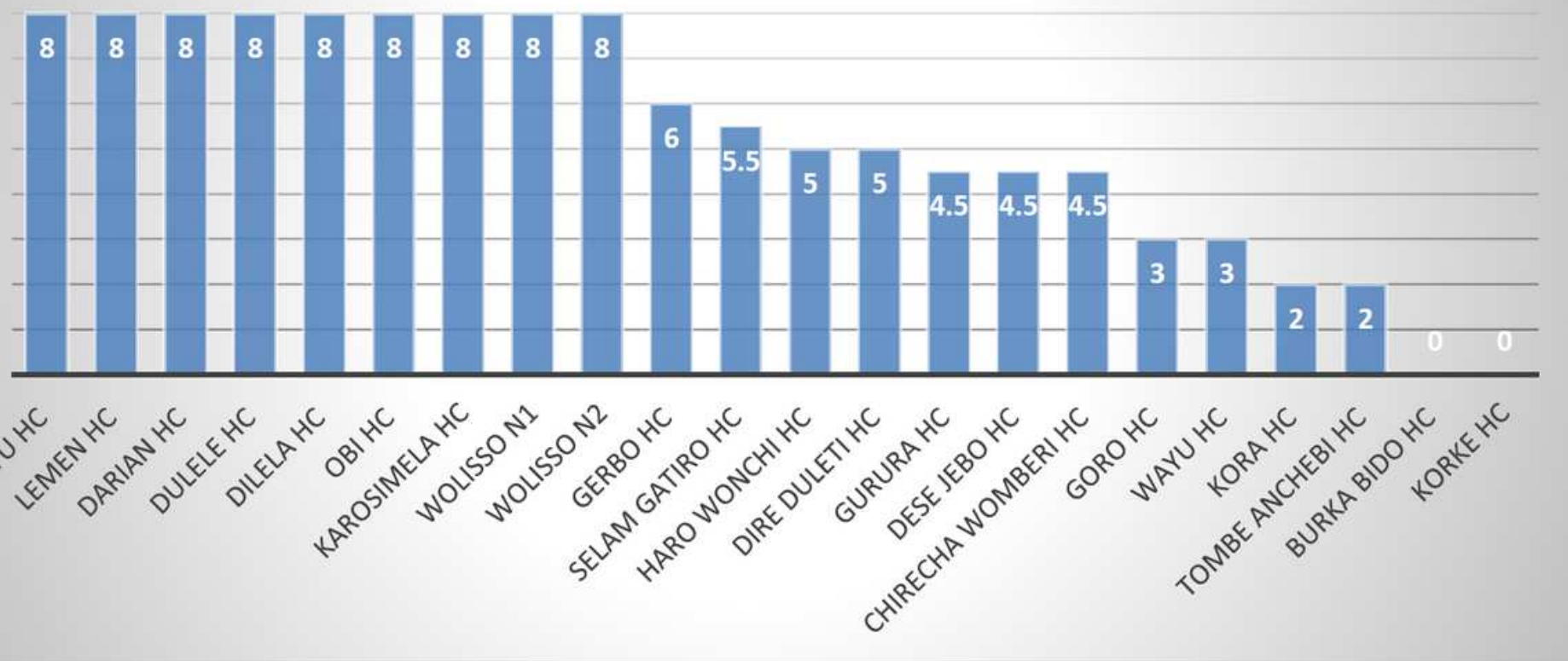

Figure 5

Supportive Supervision at Health Centers, 2019

\section{Overall Implementation of HMIS}

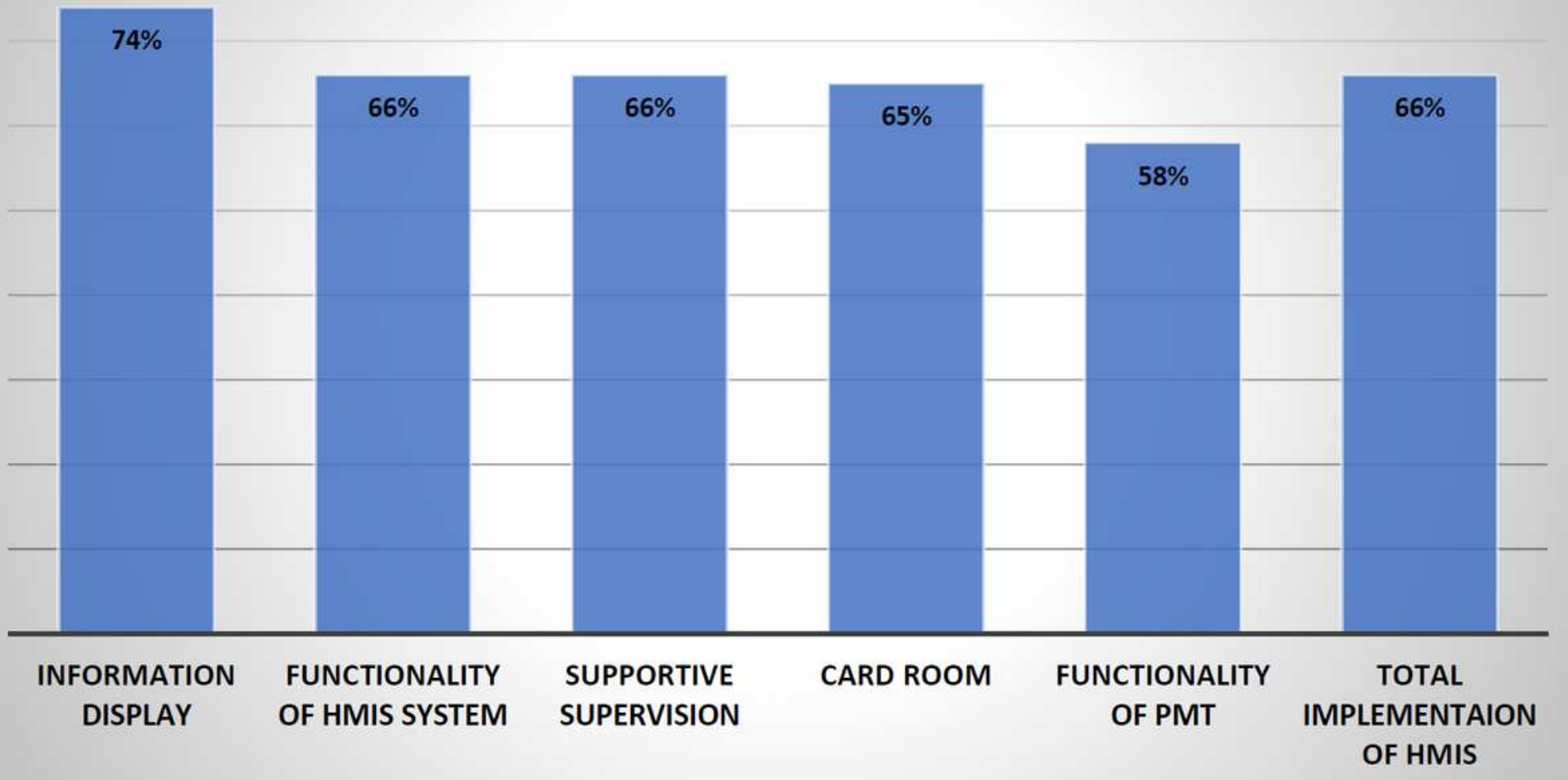

Figure 6 
Overall implementation of HMIS, 2019

\section{Supplementary Files}

This is a list of supplementary files associated with this preprint. Click to download.

- QuestionnaireforAssesmentofHMISImplementation.pdf 